\title{
Relaxação Lagrangeana Aplicada ao Problema de Cobertura por Hubs
}

\author{
C.H. SAMORA ${ }^{1}$, F.L. USBERTI ${ }^{2}$ e C. LYRA $^{1}$ \\ Recebido em 08 de Novembro, 2016 / Aceito em 19 de Junho, 2018
}

\begin{abstract}
RESUMO. Este trabalho propõe novas formulações para o problema de cobertura por hubs capacitado de alocação única com custo fixo. O problema envolve a localização de nós hubs e a atribuição de nós de demandas aos hubs, de modo que o tempo de percurso entre qualquer par de nós origem-destino não exceda a janela máxima de tempo e a capacidade de processamento dos hubs. Uma relaxação Lagrangeana é proposta e através do método do subgradiente são obtidos limitantes primais e duais. Para acelerar o método subgradiente, uma heurística construtiva primal fornece boas soluções de partida. Além disso, foi realizada uma etapa de pré-processamento das instâncias para a redução do espaço de busca. Experimentos computacionais foram realizados com um conjunto de instâncias reais da "Australian Post". Os resultados indicam que a relaxação lagrangeana proposta, quando comparada com a solução de um dos modelos de referência da literatura, foi capaz de aprimorar os limitantes primais e duais sob limites de tempo de execução e de consumo de memória.
\end{abstract}

Palavras-chave: Problema de localização de hubs, Relaxação Lagrangeana, Heurística Construtiva

\section{INTRODUÇÃO}

O Problema de localização de hubs surge no contexto de projetos de redes para o transporte de grandes quantidades de bens (passageiros, cargas, dados) entre pares origem-destino (OD). Em aplicações reais, o transporte de bens da origem direto para o destino (sem intermediações) é normalmente inviável, devido aos custos logísticos que essa política de transporte implicaria. Como alternativa, existe a rede logística denominada hub-and-spoke [15], onde nós especiais, denominados hubs, atuam como centros de coleta, classificação, consolidação, transferência, desagregação e distribuição do fluxo de bens. Os fluxos inter-hubs se beneficiam dos ganhos de escala, proporcionando alternativas mais econômicas do que mover os bens diretamente entre nós não hubs (nós spokes). As redes hub-and-spoke consistem de duas partes: uma rede de nível $h u b$ e outra rede de nível spoke [10, 1] (Figura 1).

\footnotetext{
*Autor correspondente: Fábio Luiz Usberti - E-mail: fusberti@ic.unicamp.br

${ }^{1}$ Faculdade de Engenharia Elétrica e de Computação, UNICAMP - Universidade Estadual de Campinas, Av. CEP 13083852, Campinas, SP, Brasil. E-mail: csamora@densis.fee.unicamp.br; chrlyra@densis.fee.unicamp.br

${ }^{2}$ Instituto de Computação, UNICAMP - Universidade Estadual de Campinas, Av. CEP 13083-852, Campinas, SP, Brasil. E-mail: fusberti@ic.unicamp.br
} 


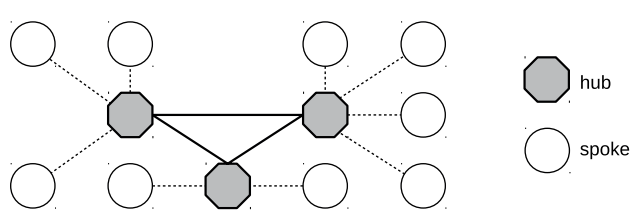

Figura 1: Hub-and-spoke de alocação única com hubs completamente conectados.

Spokes podem se conectar a um ou mais hubs, dependendo do tipo de protocolo de alocação adotado [16]. Em geral, os protocolos de alocação são dos tipos único ou múltiplo ("single assignment - SA" ou "multiple assignment - MA"). Supõe-se que as redes formadas pelos hubs são completamente conectadas [1], enquanto que os spokes não se conectam uns com os outros (ou seja, não há serviços do tipo nonstop). Portanto, todos os produtos devem passar por pelo menos um $h u b$.

Em geral, os problemas de localização de hubs lidam com dois subproblemas interdependentes: o primeiro subproblema define a localização dos hubs; o segundo subproblema define a alocação dos spokes aos hubs, de acordo com o protocolo de alocação. Quanto às complexidades desses subproblemas, tanto a localização dos hubs quanto a alocação dos spokes são problemas NPdifíceis [1].

Problemas de localização de hubs têm recebido crescente atenção na literatura [1] desde o trabalho pioneiro de O'Kelly [12, 13], em meados dos anos 80.

Mais tarde, várias formulações alternativas ao modelo apresentado por O’Kelly foram propostas na literatura $[3,6,14]$.

Campbell [3] forneceu a primeira formulação linear inteira para o problema $p$-hubs mediano, apresentou formulação matemática para o problema de localização de hubs com custo fixo e introduziu o problema de cobertura por hubs, modelado como um problema inteiro quadrático.

Kara \& Tansel [9] estudaram o problema de cobertura por hubs de atribuição única após a primeira proposição do problema por Campbell [3]. Os autores apresentaram uma formulação do problema de cobertura por hubs e mostram que o mesmo pertence à classe NP-difícil. Em seguida desenvolveram um modelo de programação não-linear inteira para o qual forneceram duas possíveis linearizações e compararam os resultados com os obtidos pelo modelo de Campbell [3].

Wagner [17] propôs uma nova formulação e uma etapa de pré-processamento que elimina todas as alocações nas quais não é possível realizar o atendimento de todos os spokes dentro de um limiar $\beta$ de tempo (ou distância) fornecido à priori. O modelo é gerado a partir de um subconjunto de atribuições válidas, considerando o limiar de tempo $\beta$ ("Valid Assignment - VA").

A contribuição deste trabalho é propor um modelo de programação linear inteira para o problema de cobertura de hubs e desenvolver uma metodologia de solução baseada em relaxação lagrangeana $[15,5]$. Na literatura, o problema investigado denomina-se "Capacitated Single Allocation 
Hub Covering with Fixed Cost Problem", ou simplesmente CHC. O problema consiste em estabelecer uma rede hub-and-spoke que minimize o custo de instalação dos hubs, sob restrições de atendimento dos fluxos de bens entre os pares origem-destino, dentro de uma janela de tempo $\beta$, sem exceder a capacidade de processamentos dos hubs. O problema tem aplicações importantes, por exemplo, para o transporte aéreo de passageiros, quando se procura reduzir os custos para as companhias aéreas e ao mesmo tempo manter o fator de satisfação dos consumidores elevado $[3,16,2,10]$.

Um aspecto inovador a destacar na abordagem proposta é a introdução no modelo matemático da restrição de capacidade de processamento dos hubs. O trabalho apresenta um modelo de quatro índices para o problema e desenvolve uma técnica de decomposição por relaxação lagrangeana.

A próxima seção apresenta a modelagem matemática para o CHC. A Seção 3 apresenta uma heurística primal, técnicas de pré-processamento e uma relaxação lagrangeana que compõem a metodologia proposta neste trabalho. Os resultados de desempenho computacional são apresentados na Seção 4. A Seção 5 apresenta as conclusões, uma breve discussão dos resultados e propõe desdobramentos das pesquisas.

\section{MODELO MATEMÁTICO}

Seja $G(N, E)$ um grafo, onde $N=\{1, \ldots, n\}$ é o conjunto de nós, tal que cada nó é uma possível localização de um $h u b ; O_{i}$ denota o fluxo do nó origem $i ; b_{i}$ denota a capacidade de processamento do nó $i$ se ele for escolhido como um nó $h u b ; f_{i}$ denota o custo fixo de instalação do $h u b$ em $i$; $t_{i k}$ é a distância/tempo entre os nós $i$ e $k$ e $\alpha$ é um fator de desconto aplicada para as distâncias entre hubs. Considera-se que as distâncias respeitam a desigualdade triangular.

A seguir é apresentado um modelo com variáveis de decisão binárias, onde $x_{i j k m}=1$ se e somente se existe um fluxo passando pelos nós $i, j, k, m$, nessa ordem, $z_{i k}=1$ se e somente se o nó $i$ foi atribuído ao hub $k(i \neq k)$ e $z_{k k}=1$ se e somente se o nó $k$ foi selecionado para ser hub. Cabe notar que as variáveis $x_{i j k m}$ são descritas por quatro índices $\left(O\left(n^{4}\right)\right)$; por esse motivo denomina-se modelo de 4-índices. 


\section{Modelo de 4-índices}

$$
\begin{array}{lr}
z= & \min \quad \sum_{k} f_{k} z_{k k} \\
\text { s.a. } & \forall i j \\
\sum_{k} \sum_{m} x_{i j k m}=1 & \forall i k \\
z_{i k} \leqslant z_{k k} & \forall i j k \\
\sum_{m} x_{i j k m}=z_{i k} & \forall i j m \\
\sum_{k} x_{i j k m}=z_{j m} & \forall k \\
\sum_{i} O_{i} z_{i k} \leqslant b_{k} z_{k k} & \forall i j k m \\
\left(t_{i k}+\alpha t_{k m}+t_{m j}\right) \cdot x_{i j k m} \leqslant \beta & \forall i j k m \\
& x_{i j k m}, z_{i k} \in\{0,1\}
\end{array}
$$

A restrição (2) garante a alocação de cada par de spokes $i$ e $j$ a um único par de $h u b s, k$ e $m$. A restrição (3) impõe que nenhum nó não hub $i$ será atribuído a um nó que não seja um nó $h u b$. A restrição (4) garante que o fluxo entre qualquer par de nós $i$ e $j$ passando pelo nó $h u b k$, deve passar por algum outro nó hub $m$ não necessariamente distinto. A restrição (5) possui uma interpretação similar; fixados qualquer par de spokes $i$ e $j$ passando pelo $h u b ~ m$, o fluxo deve passar por algum outro nó $h u b k$, não necessariamente distinto. A restrição (6) assegura que o fluxo total gerado pelos nós spokes alocados ao nó hub $k$ não exceda sua capacidade de processamento. A restrição de cobertura (7) assegura que o tempo de viagem entre qualquer origem-destino $(i, j)$ não exceda uma janela de tempo $\beta$ predefinida. A função objetivo, representada pela equação (1), procura minimizar o custo fixo de instalação dos hubs.

\section{ALGORITMO DE SOLUÇÃO}

Utiliza-se a relaxação Lagrangeana [7], resolvida com o método do subgradiente [15, 14, 5], para promover uma decomposição da formulação de 4-índices.

\subsection{Limitantes Primais}

Nesta seção será descrita a metodologia heurística responsável pela obtenção de uma solução primal. Para evitar que o método do subgradiente tenha convergência lenta, é necessário uma boa estimativa $\bar{\eta}$ para a solução factível ótima $\eta^{*}$ do problema original. Quanto melhor for esta estimativa, mais rápida será a convergência [8]. Além disso, realiza-se um pré-processamento para que o espaço de soluções seja reduzido. Isso é alcançado pela eliminação de todas as alocações nas quais não é possível atender as demandas dentro do limiar $\beta$ de tempo. Assim, é possível 
restringir a busca ao subconjunto das atribuições válidas (Valid Assignment - VA). Para isso, estende-se o procedimento proposto por Wagner [17]:

$$
V A=\left\{(i, k): 2 t_{i k} \leqslant \beta, \quad t_{i k}+\alpha \cdot \max _{m}\left\{t_{k m}\right\} \leqslant \beta\right\}
$$

Pode-se obter uma redução adicional do espaço de solução através da eliminação de nós que não são capazes de processar a si mesmos $\left(O_{k}>b_{k}: \forall k \in V A\right)$; portanto, não podem ser hubs.

$$
V A^{\prime}=\left\{(\cdot, k): O_{k} \leqslant b_{k}, \forall k \in N\right\}
$$

Uma vez determinado o conjunto $V A^{\prime}$ é possível aplicar a técnica proposta por Wagner [17] para eliminar todas as atribuições inválidas (“Invalid Assignment - IA"), conforme abaixo:

$$
I A=\left\{(i, j, k, m):(i, k),(j, m) \in V A^{\prime},\left(t_{i k}+\alpha t_{k m}+t_{m j}\right)>\beta\right\}
$$

O pseudo-código da heurística primal (hPrimal) é apresentado no Algoritmo 1, onde $S$ contém os nós candidatos a hubs, o conjunto $P$ é formado pelos nós que não podem ser $h u b s$ e o conjunto $H$ contém os nós que são hubs. A Tabela 1 apresenta uma descrição dos métodos empregados

\begin{tabular}{|c|c|}
\hline Método & Descrição \\
\hline P.highestFlow() & $\begin{array}{l}\text { Retorna um elemento } p \in P \text { que possui o maior fluxo de origem }\left(O_{p} \geqslant\right. \\
\left.O_{i}: \forall i \in P \backslash\{p\}\right)\end{array}$ \\
\hline SearchHubI() & $\begin{array}{l}\text { Instala um } h u b \text { em um dos nós candidatos de } S \text {; aplica-se esse método } \\
\text { se existir um elemento } p \in P \text { cujo fluxo } O_{p} \text { seja maior que o limiar } \\
\text { threshold. }\end{array}$ \\
\hline SearchHubII() & $\begin{array}{l}\text { Instala um } h u b \text { em um dos nós candidatos de } S \text {; aplica-se esse método } \\
\text { quando a condição de ativação do método SearchHubI() não for } \\
\text { satisfeita. }\end{array}$ \\
\hline validAlloc() & $\begin{array}{l}\text { Implementa a técnica proposta por Wagner [17] para eliminar todas as } \\
\text { atribuições inválidas ("Invalid Assignment - IA.") }\end{array}$ \\
\hline relax(threshold) & $\begin{array}{l}\text { O limiar threshold adotado para a condição de ativação do método } \\
\text { SearchHubI() é relaxado, propiciando uma busca menos gulosa para } \\
\text { instâncias mais difíceis. }\end{array}$ \\
\hline tryAllocElement() & $\begin{array}{l}\text { Resolve um problema da mochila } 0-1 \text { que consiste na atribuição de nós } \\
\text { aos hubs, minimizando suas capacidades ociosas. }\end{array}$ \\
\hline tryAllocElementOfP() & $\begin{array}{l}\text { Resolve um problema da mochila 0-1 que consiste na atribuição de nós } \\
\text { do conjunto } P \text { aos hubs, minimizando suas capacidades ociosas. }\end{array}$ \\
\hline factivel $(\bar{\eta})$ & Verifica a factibilidade primal da solução $\bar{\eta}$. \\
\hline
\end{tabular}
pelo Algoritmo 1.

Tabela 1: Métodos utilizados pelo Algoritmo 1. 


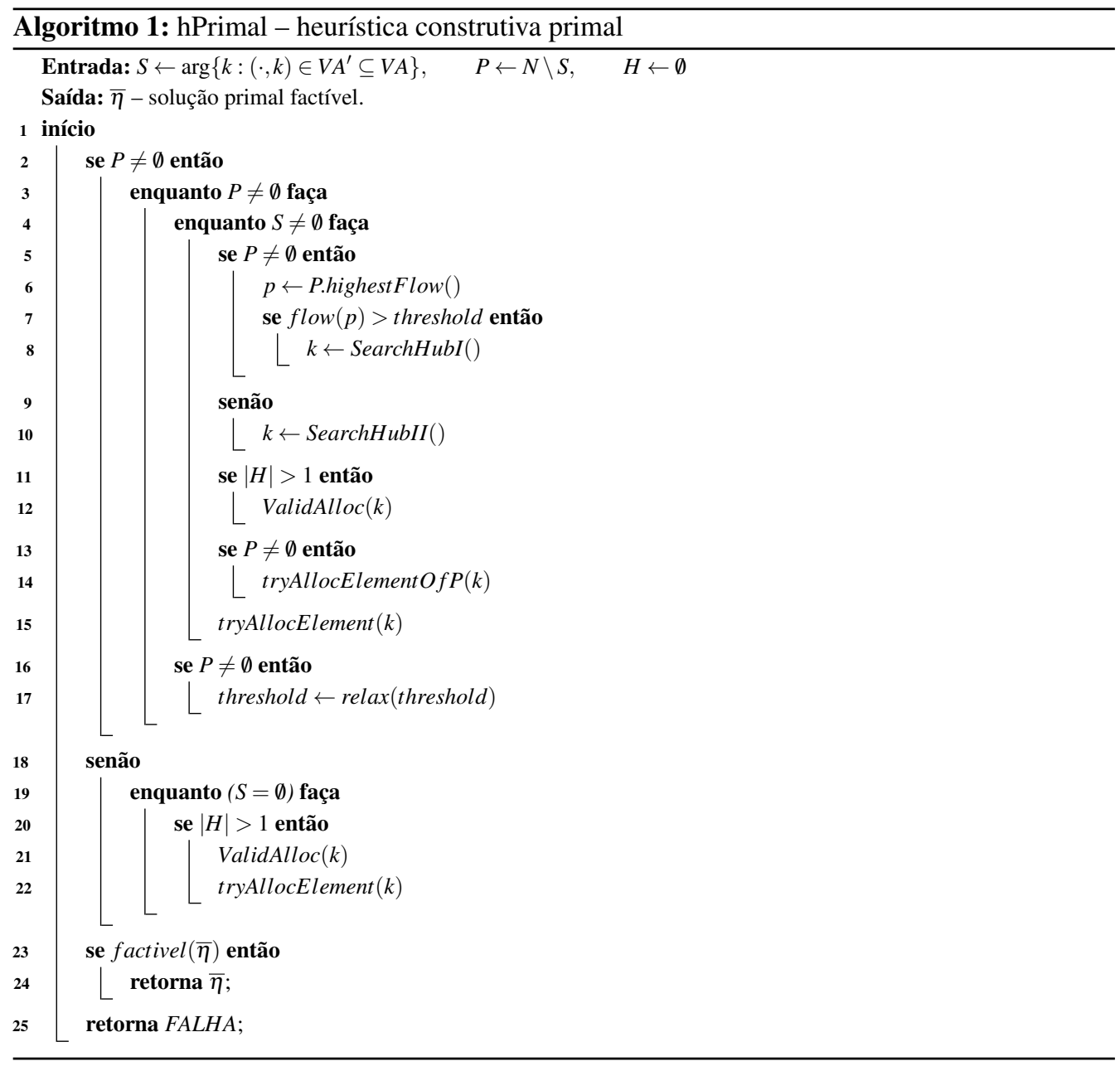

\subsection{Limitantes Duais}

Dualizando-se as restrições (4) e (5), e utilizando-se as variáveis duais $u \in \mathbb{R}^{3}$ e $v \in \mathbb{R}$, tem-se:

$$
L(u, v)=\min \sum_{k} f_{k} z_{k k}-\sum_{i, k} z_{i k} \sum_{j}\left(u_{i j k}+v_{j i k}\right)+\sum_{i, j, k, m}\left(u_{i j k}+v_{i j m}\right) \cdot x_{i j k m}
$$

Rearranjando-se os termos da função $L(u, v)$ pode-se separar a função lagrangeana em duas funções $L_{x}$ e $L_{z}$ :

$$
L(u, v)=L_{z}(u, v)+L_{x}(u, v)
$$


onde,

$$
L_{z}(u, v)=\min \quad \sum_{k} f_{k} z_{k k}-\sum_{i, k} z_{i k} \sum_{j}\left(u_{i j k}+v_{j i k}\right)
$$

s.a.

$$
\begin{array}{lc}
z_{i k} \leqslant z_{k k} & \forall i k \\
\sum_{i} O_{i} z_{i k} \leqslant b_{k} z_{k k} & \forall k \\
z_{i k} \in\{0,1\} & \forall i k
\end{array}
$$

e

$$
L_{x}(u, v)=\min \sum_{i, j, k, m}\left(u_{i j k}+v_{i j m}\right) \cdot x_{i j k m}
$$

s.a.

$$
\begin{array}{lr}
\sum_{k} \sum_{m} x_{i j k m}=1 & \forall i j \\
\left(t_{i k}+\alpha t_{k m}+t_{m j}\right) \cdot x_{i j k m} \leqslant \beta & \forall i j k m \\
x_{i j k m} \in\{0,1\} & \forall i j k m
\end{array}
$$

A solução do subproblema $L_{z}(u, v)$ pode ser obtida a partir da solução de uma série de $|N|+$ 1 problemas de mochila. Embora o problema da mochila esteja na classe de problemas NPdifíceis, existem algoritmos exatos pseudo-polinomiais para sua abordagem [11]. Neste trabalho, a solução das $|N|+1$ foi obtida pelo solver Gurobi.

O subproblema $L_{x}(u, v)$ pode ser descrito como uma família de $|N|^{2}$ subproblemas de semiassignment com uma restrição de cobertura (7). É possível tratar de modo exato o subproblema $L_{x}(u, v)$ por inspeção, conforme mostrado a seguir:

$$
x_{i j w l}=1 \Leftrightarrow \bar{c}_{i j w l}=\min \left\{u_{i j k}+v_{i j m}: k, m \in N\right\} \wedge\left\{\left(t_{i k}+\alpha t_{k m}+t_{m j}\right) \cdot x_{i j w l} \leqslant \beta\right\}
$$

Para obter um limitante dual, pode-se resolver o dual Lagrangeano do problema original [7], dado por:

$$
z_{d}=\max \quad L(u, v)
$$

\subsection{Método do Subgradiente}

O método do subgradiente é um método de otimização iterativo que encontra uma sequência de valores que convergem para o ótimo de uma função convexa [8]. Neste trabalho, a solução do problema dual Lagrangeano (23) é realizada através do método do subgradiente descrito no Algoritmo 2.

A solução do problema dual Lagrangeano retorna um limitante inferior (solução dual) $z_{d}$. A heurística hPrimal fornece o melhor limitante superior $\bar{\eta}$ para o valor ótimo do problema original; eventualmente, o algoritmo subgradiente pode melhorar a solução primal. O parâmetro $\lambda^{k}$ é 


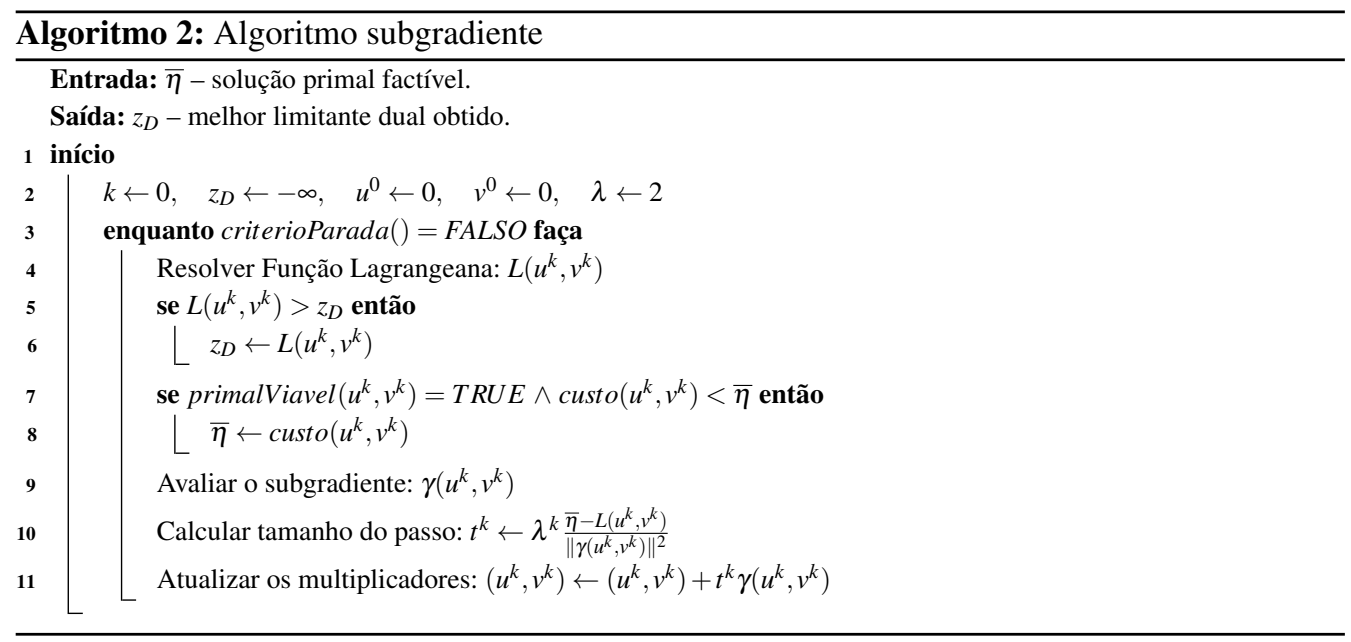

dividido à metade a cada 25 iterações consecutivas sem melhoria do limitante inferior; enquanto houver melhoria, seu valor é mantido [8].

\section{RESULTADOS COMPUTACIONAIS}

Os testes computacionais foram executados em um computador com memória de 32 GB de RAM e processador Intel @ Core i7-4790 CPU @ 3.60 GHz com 4 núcleos e 8 processadores. O solver de programação linear utilizado foi o Gurobi 6.5.

Adotou-se para os testes computacionais o conjunto de dados AP ("Australian Post"), introduzido na literatura por Ernst \& Krishnamoorthy [6]. Esse conjunto de dados foi obtido do serviço postal de 200 cidades australianas e encontra-se disponível no repositório OR-Library ${ }^{1}$.

Foram realizados testes computacionais com 20 instâncias do conjunto AP, contendo de 10 a 50 vértices, e parâmetros nos intervalos $0,2 \leqslant \alpha \leqslant 0,8$ e $35.000 \leqslant \beta \leqslant 60.000$. O tempo de execução do solver Gurobi foi limitado em 3 horas. A condição de parada para o método do subgradiente foi de 15 minutos, ou um máximo de 80 iterações, ou tamanho do passo $\left(\lambda_{k}\right)$ menor que 0,001 .

A Tabela 2 apresenta os resultados computacionais obtidos. A primeira coluna apresenta a instância. Em seguida, tem-se as colunas correspondentes à Relaxação Lagrangeana e as colunas correspondentes à solução do modelo de 4-índices pelo solver Gurobi. Para cada metodologia, foram incluídas as colunas "Primal", "Dual", "gap" e "tempo", que fornecem as melhores soluções primal e dual obtidas, o gap de otimalidade e o tempo de execução.

Analisando-se a Tabela 2 é possível observar que a relaxação lagrangeana não atingiu a solução ótima para 12 instâncias, enquanto que a solução do modelo de 4-índices não atingiu a solução ótima para 5 instâncias. No entanto, o desempenho do modelo de 4-índices para essas cinco

\footnotetext{
${ }^{1}$ http://people.brunel.ac.uk/ mastjjb/jeb/orlib/phubinfo.html
} 
Tabela 2: Resultados dos experimentos computacionais.

\begin{tabular}{|c|c|c|c|c|c|c|c|c|}
\hline \multirow{2}{*}{$\begin{array}{l}\text { Instância } \\
(n ; \alpha ; \beta)\end{array}$} & \multicolumn{4}{|c|}{ Relaxação Lagrangeana } & \multicolumn{4}{|c|}{ Modelo 4-índices } \\
\hline & Primal & Dual & gap & tempo $(s)$ & Primal & Dual & gap & tempo $(s)$ \\
\hline$(10 ; 0,2 ; 35 \mathrm{k})$ & $77.785,96$ & $77.551,71$ & 0,3 & 0,3 & $77.785,96$ & $77.785,96$ & 0,0 & 0,4 \\
\hline$(10 ; 0,4 ; 35 \mathrm{k})$ & $82.996,96$ & $78.456,77$ & 5,8 & 0,3 & $78.921,53$ & $78.921,53$ & 0,0 & 0,4 \\
\hline$(10 ; 0,6 ; 35 \mathrm{k})$ & $82.996,96$ & $82.996,86$ & 0,0 & 0,2 & $82.996,96$ & $82.996,96$ & 0,0 & 0,3 \\
\hline$(10 ; 0,8 ; 35 \mathrm{k})$ & $103.759,83$ & $103.758,46$ & 0,0 & 0,3 & $103.759,83$ & $103.759,84$ & 0,0 & 0,1 \\
\hline$(20 ; 0,2 ; 45 \mathrm{k})$ & $53.369,79$ & $53.369,79$ & 0,0 & 1,7 & $\mathbf{5 3 . 3 6 9 , 8 0}$ & $53.369,79$ & 0,0 & 28,9 \\
\hline$(20 ; 0,4 ; 45 \mathrm{k})$ & $70.270,08$ & $70.270,08$ & 0,0 & 1,6 & $70.270,08$ & $70.270,08$ & 0,0 & 116,9 \\
\hline$(20 ; 0,6 ; 45 \mathrm{k})$ & $70.270,08$ & $70.270,08$ & 0,0 & 1,8 & $70.270,08$ & $70.242,00$ & 0,0 & 112,3 \\
\hline$(20 ; 0,8 ; 45 \mathrm{k})$ & $70.270,08$ & $70.270,08$ & 0,0 & 1,7 & $70.270,08$ & $70.270,08$ & 0,0 & 12,9 \\
\hline$(25 ; 0,2 ; 50 \mathrm{k})$ & $45.271,28$ & $43.077,32$ & 5,1 & 3,8 & $43.194,26$ & $43.194,26$ & 0,0 & 254,9 \\
\hline$(25 ; 0,4 ; 50 \mathrm{k})$ & $67.328,90$ & $63.297,12$ & 6,4 & 3,9 & $65.490,87$ & $65.490,87$ & 0,0 & $1.611,0$ \\
\hline$(25 ; 0,6 ; 50 \mathrm{k})$ & $71.765,38$ & $66.562,78$ & 7,8 & 2,8 & $67.183,10$ & $67.183,10$ & 0,0 & 639,0 \\
\hline$(25 ; 0,8 ; 50 \mathrm{k})$ & $75.942,83$ & $75.489,85$ & 0,6 & 1,6 & $75.942,83$ & $75.942,83$ & 0,0 & 79,0 \\
\hline$(40 ; 0,2 ; 55 \mathrm{k})$ & $67.901,53$ & $61.106,17$ & 11,1 & 503,1 & 63.567,55 & $63.567,55$ & 0,0 & $3.226,5$ \\
\hline$(40 ; 0,4 ; 55 \mathrm{k})$ & $74.473,88$ & $68.389,15$ & 8,9 & 320,0 & $69.590,09$ & $69.590,09$ & 0,0 & $5.316,9$ \\
\hline$(40 ; 0,6 ; 55 \mathrm{k})$ & $86.897,15$ & $84.797,54$ & 2,5 & 278,9 & $96.909,75$ & $78.293,78$ & 23,8 & $10.800,4$ \\
\hline$(40 ; 0,8 ; 55 \mathrm{k})$ & $118.483,28$ & $117.986,35$ & 0,4 & 167,3 & $118.483,29$ & $118.483,29$ & 0,0 & $2.765,6$ \\
\hline$(50 ; 0,2 ; 60 \mathrm{k})$ & $52.699,59$ & $52.699,59$ & 0,0 & 380,2 & $89.598,17$ & $51.605,02$ & 73,6 & $10.858,7$ \\
\hline$(50 ; 0,4 ; 60 \mathrm{k})$ & $54.510,48$ & $54.510,48$ & 0,0 & 589,4 & $194.027,20$ & - & - & $10.800,7$ \\
\hline$(50 ; 0,6 ; 60 \mathrm{k})$ & $71.002,66$ & $69.837,58$ & 1,7 & 603,2 & $231.382,64$ & - & - & $10.801,4$ \\
\hline$(50 ; 0,8 ; 60 \mathrm{k})$ & $96.544,53$ & $87.528,39$ & 10,3 & 654,8 & $191.436,01$ & $74.154,02$ & 158,2 & $10.820,5$ \\
\hline
\end{tabular}

gap - desvio de otimalidade (\%)

instâncias foi bastante insatisfatório: para duas delas, $(50 ; 0,4 ; 60 k)$ e $(50 ; 0,6 ; 60 k)$, o modelo de 4-índices não conseguiu sequer encontrar um limitante dual, enquanto que a relaxação lagrangeana obteve bons limitantes duais para todas as instâncias. Para as outras três instâncias, o modelo de 4-índices obteve um gap de otimalidade de $85,2 \%$, enquanto que a relaxação lagrangeana, para essas mesmas instâncias, obteve um gap de otimalidade de 4,3\%. Considerando-se todas as instâncias, o gap de otimalidade médio foi de 14,2\% e 3,0\% para o modelo de 4-índices e para a relaxação lagrangeana, respectivamente. Cabe observar também que os limitantes primais obtidos pela relaxação lagrangeana foram substancialmente melhores dos que os obtidos com o modelo de 4-índices, apresentando uma redução média de 21,9\%. Finalmente, a relaxação lagrangeana atingiu a solução ótima para duas instâncias, $(50 ; 0,2 ; 60 k)$ e $(50 ; 0,4 ; 60 k)$, para as quais o modelo de 4-índices não conseguiu determinar o ótimo.

O bom desempenho da relaxação lagrangeana em termos de tempo computacional se deve, em parte, à qualidade da estimativa obtida pela heurística primal, fornecida ao método do subgradiente. De fato, das onze soluções ótimas identificadas pela relaxação lagrangeana, seis delas já haviam sido obtidas pela heurística primal.

A Figura 2 apresenta o consumo de memória por tamanho de instância para a relaxação lagrangeana e para o modelo de 4-índices. Percebe-se que o consumo de memória do modelo de 4-índices cresce em taxa muito maior que a relaxação lagrangeana. Portanto o método subgradiente tem potencial de resolver instâncias com dimensões maiores, decorrentes de problemas reais. 


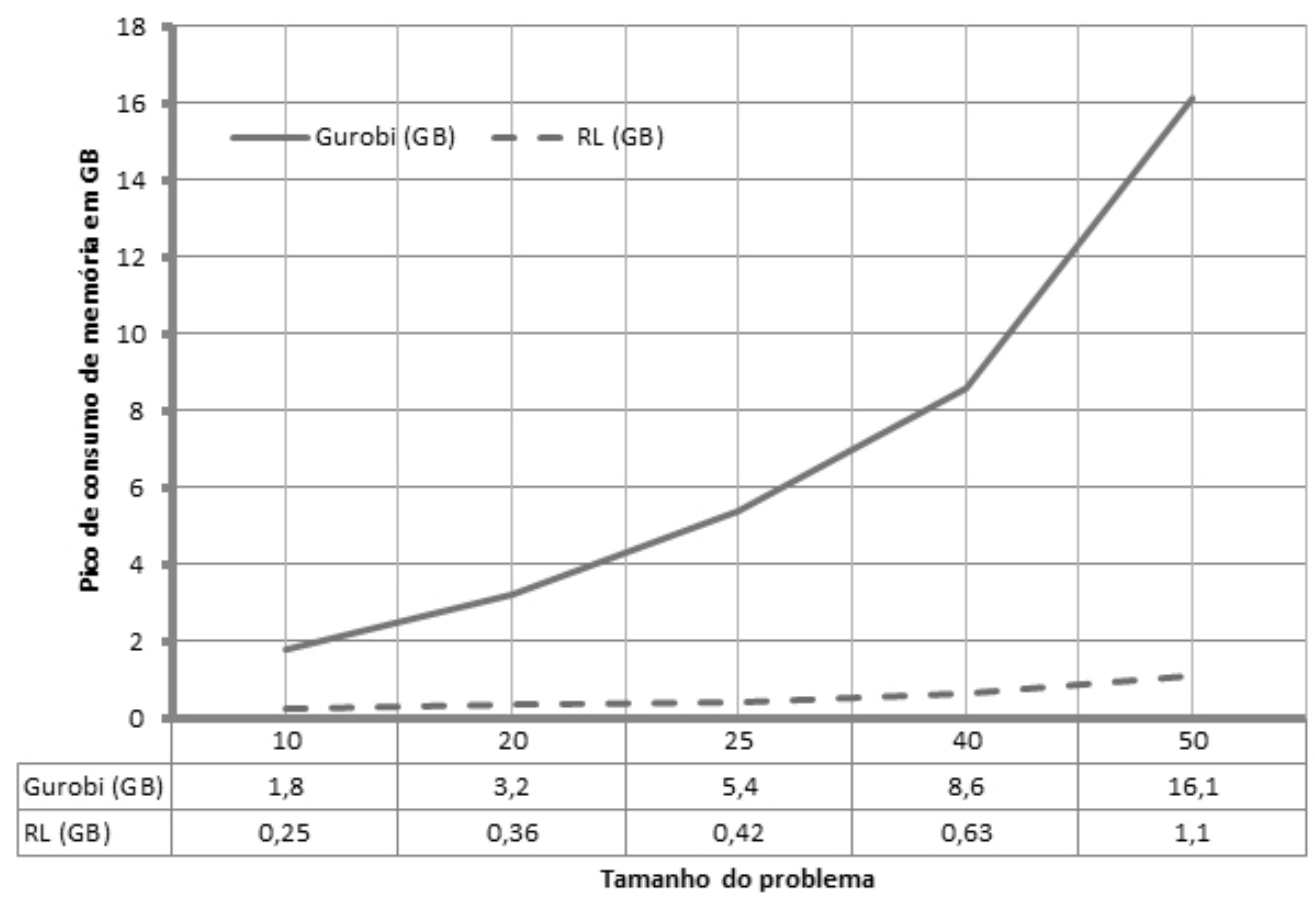

Figura 2: Consumos de memória pelo Gurobi e pelo método do subgradiente.

\section{COMENTÁRIOS FINAIS}

Neste trabalho, foram elaborados estudos de modelagem para o problema de cobertura de hubs, originalmente proposto por Campbell [3]. O modelo de 4-índices da literatura foi decomposto em dois subproblemas a partir de uma relaxação lagrangeana.

Uma fase de pré-processamento, com redução significativa do espaço de busca, foi efetiva para diminuir o esforço computacional. Por outro lado, a heurística construtiva proposta foi capaz de fornecer bons limitantes primais e, em alguns casos, atingir a solução ótima.

As soluções primais obtidas com o emprego da relaxação Lagrangenana foram melhores do que as soluções obtidas com a solução do modelo de 4-índices. De fato, para as maiores instâncias o modelo de 4-índices, resolvido com o Gurobi, encontrou dificuldades de convergência; para duas instâncias, o modelo não conseguiu sequer encontrar um limitante dual. A relaxação lagrangeana também apresentou um gap de otimalidade médio melhor e um consumo de memória computacional menor do que o modelo de 4-índices. Isto permite concluir que a relaxação lagrangeana é um método promissor para o tratamento de instâncias de maior porte. 


\title{
ACKNOWLEGMENTS
}

Este trabalho teve o apoio do CNPq (proc. 132601/2013-3, 307472/2015-9, 425340/2016-3) e FAPESP (proc. 2015/11937-9).

\begin{abstract}
This paper proposes new formulations for the single allocation fixed-cost hub covering problem. This problem concerns on determining the location of hubs and the assignment of demand nodes to hubs, respecting the hubs capacities and maintaining the traversal time between any pair of nodes within a time-window. A Lagrangean relaxation is proposed and through the subgradient method, lower and upper bounds are obtained. To improve the method performance, a primal constructive heuristic obtains good warm-start solutions. In addition, a pre-processing reduces the solution space. Computational experiments were conducted with a set of real-life instances from the Australian Post. The results indicate that the proposed Lagrangean relaxation, when compared with the solution of reference model from literature, was capable of improving the upper and lower bounds, under restrictions on the execution time and memory usage.
\end{abstract}

Keywords: Hub covering problem, Lagrangean relaxation, Constructive heuristics.

\section{REFERÊNCIAS}

[1] S. Alumur \& Kara, B. Y. Network hub location problem: the state of the art. European Journal of Operational Research , 190 (1) (2008), 1-21.

[2] D. L. Bryan \& M. E. O'Kelly. Hub-and-spoke network in air transportation: an analytical review. Journal of Regional Science , 39 (2) (1999), 275-295.

[3] J. F. Campbell , Integer programming formulation of discrete hub location problems. European Journal of Operational Research , 72 (1994b), 387-405.

[4] J. F. Campbell \& Morton E. O'Kelly. Twenty-five years of hub location research. Transportation Science, 46 (2012), 153-169.

[5] I. Contreras, J. A. Díaz \& E. Fernández. A Lagrangean relaxation approach for the capacitated single allocation hub location problem. "Meeting of the thematic network: Analysis and applications decisions on locations of services and related problems", Baeza, Spain, Mars (2007).

[6] A. T. Ernst \& M. Krishnamoorthy, Efficient algorithms for the uncapacitated single allocation p-hub median problem. Location Science, 4 (3) (1996b), 139-154.

[7] A. M. Geoffrion, The Lagrangian relaxation method for solving integer programming problems. Management Science, 27 (1) (1974), 1-18.

[8] J. L. Goffin, On convergence rate of subgradient optimization methods. Mathematical Programming, 13 (1977), 329-347. 
[9] B. Y. Kara \& B. C. Tansel, The single-assignment hub covering problem: models and linearizations. Journal of the Operational Research Society, 54 (2003), 59-64.

[10] J. G. Klincewicz, Hub location in backbone tributary network design: a review. Location Science, 6 (1998), 307-335.

[11] S. Martello, D. Pisinger \& P. Toth., Dynamic programming and strong bounds for the 0-1 knapsack problem. Management Science, 45 (3) (1999) INFORMS.

[12] M. E. O'Kelly, The location of interacting hub facilities. Transportation Science, 20 (1986), 92-106.

[13] M. E. O'Kelly, A quadratic integer programming for location of interacting hub facilities. European Journal of Operational Research, 32 (1987), 393-404.

[14] D. Skorin-Kapov, J. Skorin-Kapov \& M. E. O’Kelly, Tight linear programming relaxations of uncapacitated p-hub median problems. European Journal of Operation Research, 94 (1996a), 582-593.

[15] A. Turgut, Lagrangian relaxation based approaches to capacitated hub-and-spoke network design problem. European Journal of Operational Research, 79 (1994), 501-5023.

[16] A. Turgut, Networking policies for hub-and-spoke systems with applications to the air transportation system. Transportation Science, 3 (1995), 201-221.

[17] B. Wagner, Model formulation for hub covering problems. Journal of the Operational Research Society, 59 (7) (2008), 932-938. 